\title{
Large optical cavity AIGaAs buried heterostructure window lasers
}

\author{
H. Blauvelt, S. Margalit, and A. Yariv \\ California Institute of Technology, Pasadena, California 91125
}

(Received 4 January 1982; accepted for publication 29 March 1982)

Large optical cavity buried heterostructure window lasers in which only the transparent AlGaAs waveguiding layers, and not the active layer, extend to the laser mirrors have been fabricated. These lasers have threshold currents and differential quantum efficiencies comparable to those of regular large optical cavity buried heterostructure lasers in which the active region extends to the laser mirrors; however, the window lasers have been operated under pulsed conditions at three times the power at which otherwise identical lasers without windows degrade by catastrophic mirror damage.

PACS numbers: 42.55.Px, 42.80.Sa

Many failure mechanisms of $\mathrm{AlGaAs}$ lasers can be attributed to the presence of the active layer at the laser mirrors. Local heating due to optical absorption in the active layer near the mirrors can result in catastrophic mirror damage at high output powers. At somewhat lower output powers, facet erosion due to oxidation of the active layer can occur. Even at low output powers, defects are generated at a fast rate in the active region in the vicinity of mirrors. Catastrophic mirror damage can be avoided by making the laser structure transparent to the light output in the vicinity of the mirrors. This has been accomplished previously by selective $\mathrm{Zn}$ diffusion in stripe lasers and results in an order of magnitude increase in the available pulsed optical power. ${ }^{1}$ The power output of $\mathrm{Zn}$ diffused window lasers is limited by catastrophic damage due to local heating in the bulk rather than at the laser mirrors. There is also evidence that laser degradation due to facet oxidation is greatly reduced in lasers with transparent AlGaAs mirrors. ${ }^{2}$ This has been attributed to the greater stability of the native oxide of $\mathrm{AlGaAs}$ compared

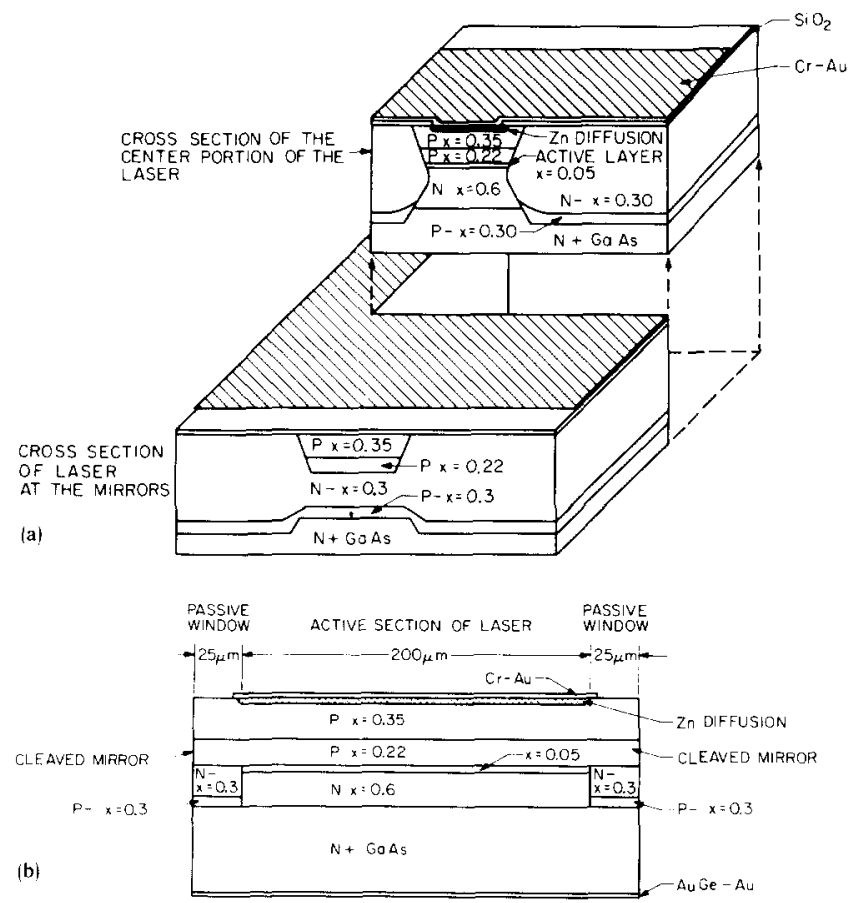

FIG. 1. (a) Schematic diagram of a window LOC BH laser structure. (b) Side view of a window LOC BH laser. to the native oxide of GaAs.

In this letter we report on large optical cavity (LOC) buried heterostructure (BH) window lasers in which only the transparent AlGaAs layers, and not the active layer, extend to the laser mirrors. These layers have threshold currents and quantum efficiencies comparable to those of LOC BH lasers without transparent end sections and have been operated at up to three times the power at which regular LOC BH lasers degrade by catastrophic mirror damage. Window LOC BH lasers also appear to be more resistant to degradation due to mirror oxidation.

The window LOC BH laser structure is illustrated in Fig. 1. The laser actually consists of two separate structures, a $200-\mu \mathrm{m}$ central section which is a regular LOC BH structure ${ }^{3}$ grown by liquid phase epitaxy (LPE) and $25-\mu \mathrm{m}$ transparent window sections from which the active layer and lower cladding layers have been removed after the first growth

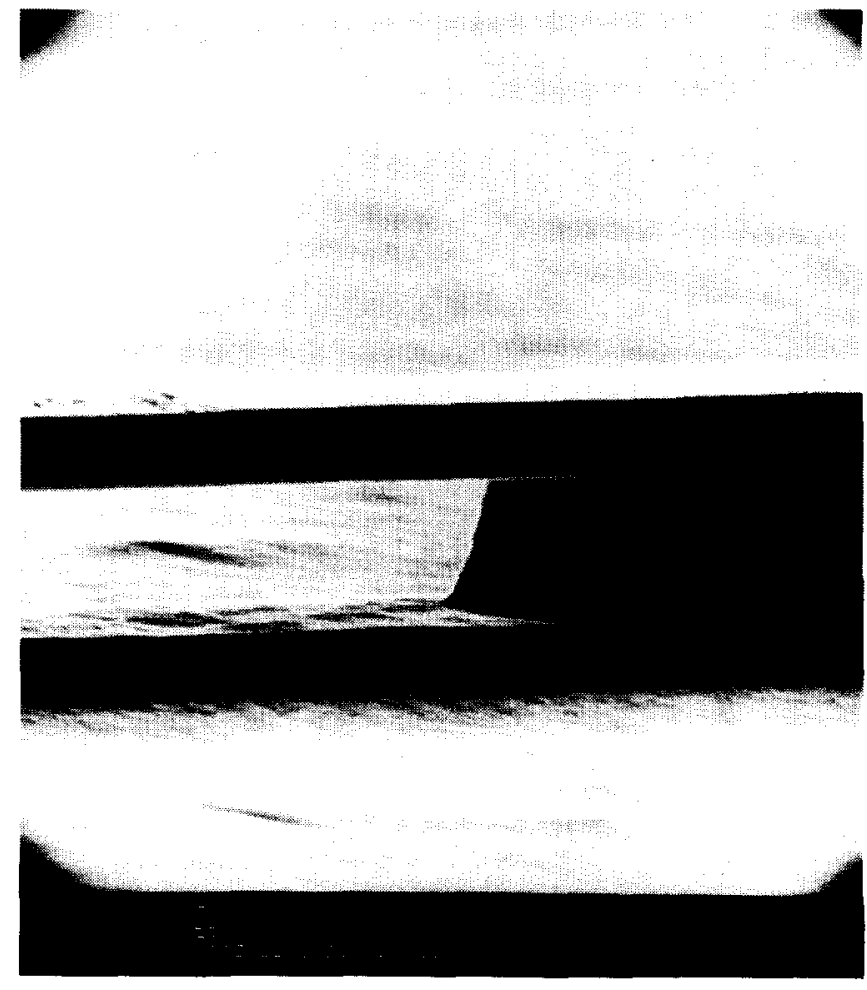

FIG. 2. SEM photograph of a selectively etched mesa prior to a second LPE growth. 

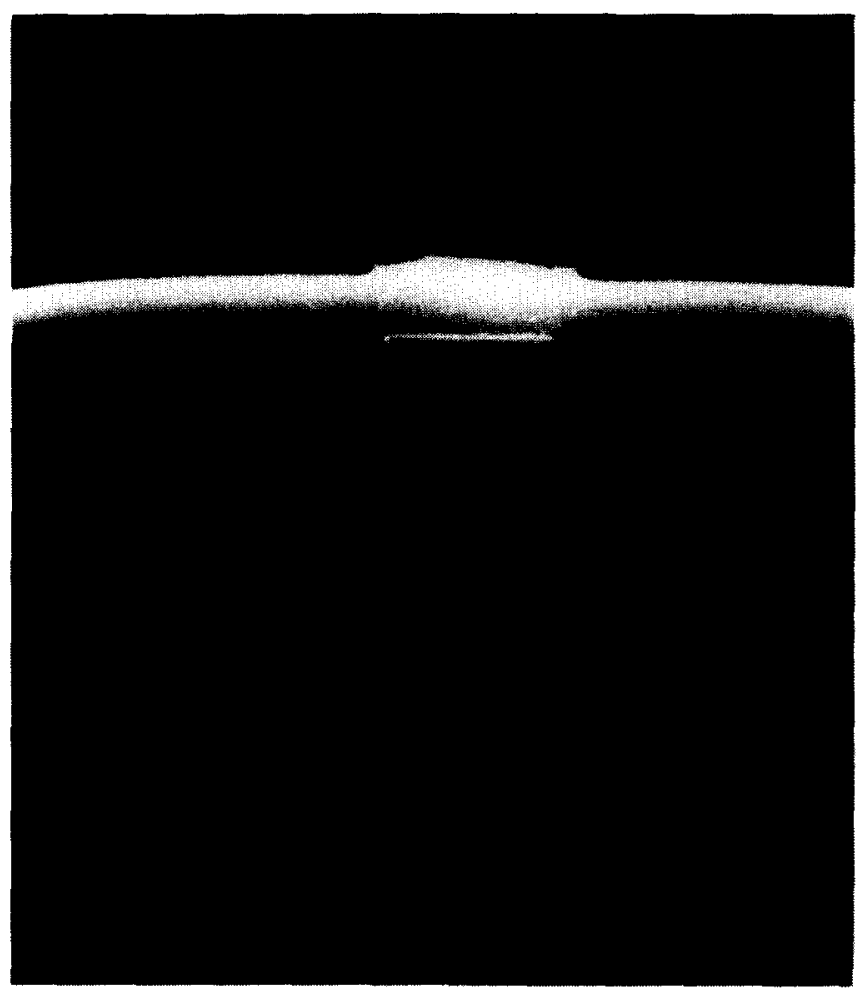

FIG. 3. SEM photograph of the cross section of a window section of a LOC BH laser.

and replaced by transparent $\mathrm{AlGaAs}$ layers that are added during a second LPE growth. The optical mode is mostly confined to the $\mathrm{Al}_{0.22} \mathrm{Ga}_{0.78}$ As guide layer in both the center and window sections, which results in low loss coupling of the laser mode between the waveguides in the center and window sections.

The fabrication of the window LOC BH laser requires two LPE growths. The layers grown in the first growth are $n$ -

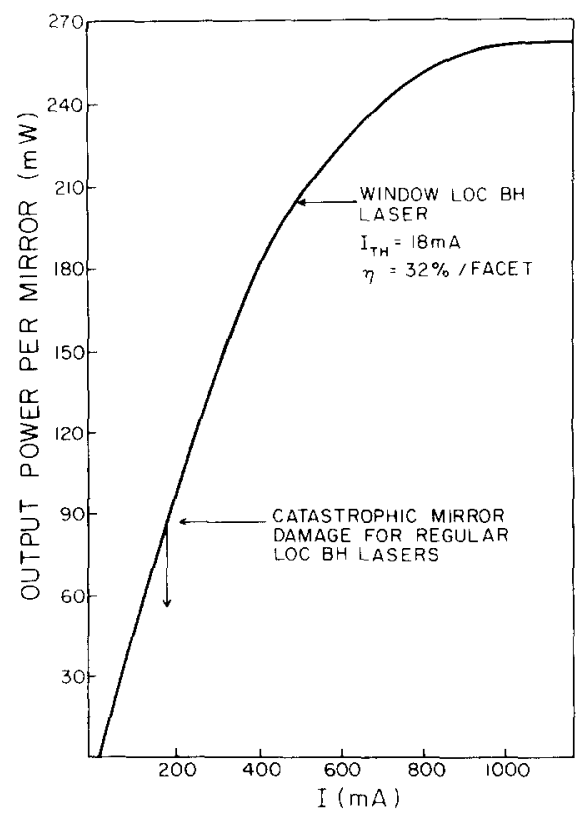

FIG. 4. Light vs current characteristics of a regular and window LOC BH laser
$\mathrm{Al}_{0.6} \mathrm{Ga}_{0.4} \mathrm{As}$ (1.5 $\mu \mathrm{m}$, Te doped), undoped $\mathrm{Al}_{0.05} \mathrm{Ga}_{0.95} \mathrm{As}$ $(0.12 \mu), p-\mathrm{Al}_{0.22} \mathrm{Ga}_{0.78}$ As $(0.7 \mu \mathrm{m}, \mathrm{Ge}$ doped), and $p$ $\mathrm{Al}_{0.35} \mathrm{Ga}_{0.65} \mathrm{As}(1.0 \mu \mathrm{m}, \mathrm{Ge}$ doped). Next, mesas are etched as is done for a regular $\mathrm{BH}$ laser. The mesas are then masked by photoresist, except for $50-\mu \mathrm{m}$ sections, which will eventually be the window sections of the laser. The $\mathrm{Al}_{0.6} \mathrm{Ga}_{0.4} \mathrm{As}$ in the unmasked sections is then removed by selective etching in HF. This is followed by a brief nonselective etch to remove the active region as well in the unmasked sections. Figure 2 is a scanning electron microscope (SEM) photograph of a mesa prior to the second LPE growth showing the selective removal of the bottom cladding layer in the window section. After this, a thin $p-\mathrm{Al}_{0.3} \mathrm{Ga}_{0.7}$ As layer and an $n$ $\mathrm{Al}_{0.3} \mathrm{Ga}_{0.7}$ As layer are grown in the second LPE growth. These layers grow on the sides of the unetched sections of the mesas, and both underneath and on the sides of the selectively etched sections. Figure 3 is a scanning electron micrograph of a cross section of a window section of the laser, after the second growth.

Normally, LOC BH lasers are grown with $n$-type optical guide layers to minimize electron leakage. When $n$-type GaAs substrates are used this means the active layer is on top of the guide layers. However, it is difficult to fabricate these window lasers with the active layer on top of the guide layer, since this would require growing layers over the AlGaAs guide layer in the second LPE growth. By having the active layer underneath the optical guide layer, the layers of the second growth can be grown starting from the GaAs substrate. Window LOC BH lasers can still have $n$-type optical guide layers if $p$-type GaAs substrates are used.

Since the transparent $\mathrm{Al}_{0.22} \mathrm{Ga}_{0.78}$ As guide layer, to which most of the optical power is confined, extends from mirror to mirror, there is very little coupling loss between the center and window sections of the laser. Typically, our window LOC BH lasers have threshold current of 8-10 mA per $\mu \mathrm{m}$ stripe width of the lasers for pulsed operation and 9$12 \mathrm{~mA}$ per $\mu \mathrm{m}$ for $\mathrm{cw}$ operation and differential quantum efficiencies of $20-30 \%$ per facet. The best results obtained for $2-\mu \mathrm{m}$-wide stripes were pulsed and $\mathrm{cw}$ threshold currents of 15 and $18 \mathrm{~mA}$, respectively, and a differential quantum efficiency of $35 \%$ per facet. These results are nearly identical

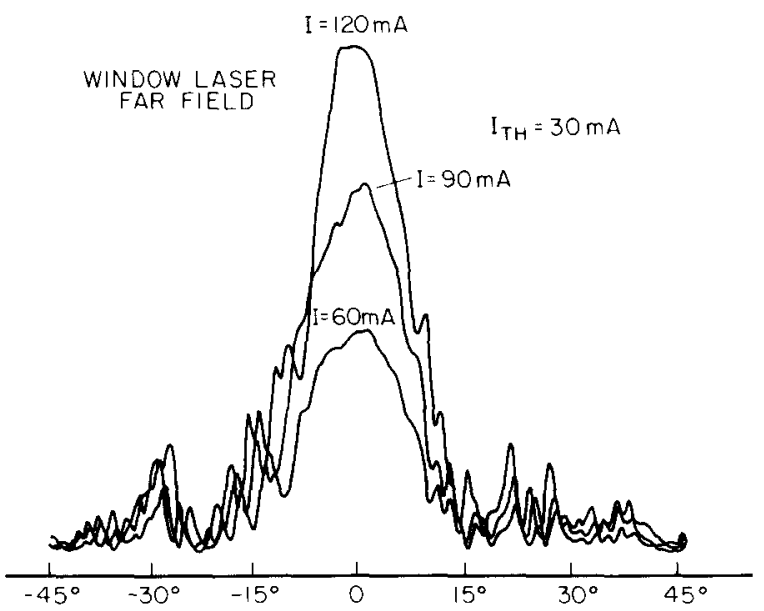

FIG. 5. Far field pattern of a window LOC BH laser. 
to those of regular LOC BH lasers fabricated from the same wafer. The most significant difference between the window and regular LOC BH lasers was the ability of the window lasers to operate at high pulsed output powers without catastrophic mirror damage. Figure 4 shows the light versus current characteristics of a LOC BH laser and a window LOC BH laser for $2-\mu \mathrm{m}$-wide devices driven by 75 -ns pulses at a repetition rate of $1 \mathrm{kHz}$. Window LOC BH lasers were operated up to $130-\mathrm{mW} / \mu \mathrm{m}$ stripe width, which is three times the power at which regular LOC BH lasers fabricated from the same wafer failed due to catastrophic mirror damage. The output power of the window lasers was limited by heating and not by mirror damage.

For stripe widths of less than $2 \mu \mathrm{m}$, operation of the window LOC BH lasers in the fundamental transverse mode can be obtained. The far fields typically had irregularities due to scattered light as is shown in Fig. 5. Irregularities in the far field typically are present in regular BH lasers as well due to scattering from the sidewalls of the laser. In window LOC BH lasers scattered light can also result from losses in the coupling of the laser light between the center and window sections. However, we were not able to determine whether coupling losses were significantly affecting the beam quality. We are currently working on other laser structures that are compatible with the window laser technology, to try to improve the laser beam quality.

We have also examined the effect of mirror oxidation on the laser performance by oxidizing the mirrors in boiling water. At regular intervals during this accelerated mirror oxidation test the pulsed laser characteristics were measured. Lasers without windows were found to degrade approximately four times as fast as window lasers. One possible explanation for this is that the oxide that is grown on the AlGaAs window sections is more stable than the oxide grown on the active layer of lasers without windows.

In conclusion, window LOC BH lasers with transparent waveguides at the mirrors have been fabricated. These lasers have threshold currents and quantum efficiencies that are nearly identical to those of regular LOC BH lasers, but have been operated at pulsed power outputs which are three times the level at which regular LOC BH lasers degrade by catastrophic mirror damage. Even greater output powers may be possible since the power is currently limited by heating of the window LOC BH lasers rather than catastrophic damage. The window lasers also appear to be less susceptible to damage due to facet oxidation.

This research was supported by the National Science Foundation (Optical Communication Program) and the Office of Naval Research.

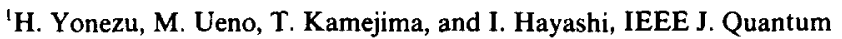
Electron. QE-15, (1979).

${ }^{2}$ S. Takahashi, T. Kobayashi, H. Saito, and Y. Furakawa, Jpn. J. Appl. Phys. 17, 865 (1978).

${ }^{3}$ N. Chinone, K. Saito, R. Ito, K. Aiki, and N. Shige, Appl. Phys. Lett. 35 $513(1979)$.

\title{
Improvements on the electrical resistivity of chemical bath deposited CdS films by laser annealing
}

\author{
G. Martínez $z^{\text {a) }}$ and J. L. Martínez \\ Instituto de Ciencias, Universidad Autónoma de Puebla, Apdo. Postal J-48, Puebla, Pue. Mexico \\ A. Zehe \\ Sektion Physik, Technische Universitaet Dresden, DDR-8027 Dresden, Mommsenstrasse 13, G. D. R.
}

(Received 5 October 1981; accepted for publication 30 March 1982)

The dark resistivity of $\mathrm{cw} \mathrm{Ar}{ }^{+}$ion laser annealed chemical bath deposited $\mathrm{CdS}$ films has been measured. A two-decade decrement of the electrical resistivity, as compared to the unannealed samples, is reported. A threshold laser power density for reproducible annealing has been found to be $>50 \mathrm{~W} / \mathrm{cm}^{2} . \log \rho$ vs $1 / T$ plots were used to calculate the mobility ratio $b=\mu_{n} / \mu_{p}$. This ratio shows a slight increment on laser annealed but it is not sufficient to explain the resistivity changes.

PACS numbers: $81.40 . \mathrm{Rs}, 79.20 . \mathrm{Ds}, 81.15 . \mathrm{Lm}, 73.60 . \mathrm{Fw}$

CdS films are deposited by vacuum evaporation, sputtering, and chemical bath deposition techniques. ${ }^{1-3}$ Generally, in each of these deposition methods polycrystalline $\mathrm{CdS}$ films are obtained and their electrical properties are very

\footnotetext{
${ }^{2}$ Present address: Elect. Engn. Dept., University of Arizona, Tuscon, AZ 85721 .
}

sensitive to the method of preparation. Thermal annealing of vacuum and chemical spray deposited $\mathrm{CdS}$ films have shown to improve their electrical characteristics in some cases by changing the substrate temperature during deposition. ${ }^{4}$ Chemical bath deposited (CBD) films show good photoconductive and electrical characteristics, however, this thinfilm deposition technique is restricted to temperatures of 20 